\title{
College English learning platform based on Android smartphone
}

\author{
Yanqiu He ${ }^{1}$, Guangyao Xiong ${ }^{2, *}$ \\ ${ }^{1}$ College of Humanities, Jiangxi University of Traditional Chinese Medicine, NanChang, 330029, China \\ ${ }^{2}$ Network Education Technology Center, JiangXi University of Traditional Chinese Medicine, \\ NanChang, 330001, China \\ *Corresponding author
}

Keywords: Multi-mode constraints, English learning platform, Online-incremental learning.

\begin{abstract}
Firstly a multi-mode binding mathematical model is provided for specific analysis on its properties. Then multi-mode constraints oriented Android smartphone based college English learning platform is proposed based on the properties of kernel function matrix and block matrix calculation formula. Application of this algorithm can achieve effective utilization of past training results, so as to effectively reduce storage and computing time. At last the validity of this learning method is verified by means of simulation experiments.
\end{abstract}

\section{Introduction}

Multi-mode constraints, abbreviate to MMC, is a new type machine learning method, proposed by Vapnik, etc[1].This method has shown its extremely powerful superiority in learning capacity and scholars have expressed strong interest. This method has achieved remarkable effect in a lot of domains such as function estimation[2]. Abovementioned elaborations are off-line cases which can be achieved by off-line type learning method. But if learning sample requires for online-collection or time sequencing, college English learning method is required such as Android smartphone based college English learning platform, online-incremental algorithm is required for realization[3].We should be aware that college English learning method has strengthened the online adaptability of learning machine, promoted and realized the time evolution ability of learning machine [4]. Thus, in recent years, a lot of scholars started to attempt studying online learning algorithm or different multi-mode constraints incremental learning algorithm [5-6].However, studies on these algorithms are realized under constraints of standard multi-mode, which means they are solution of quadric form matric issue under constraint framework, requiring for large calculated quantity and complexity. But efficient online algorithm can make sure that parameter update is timely, and effectively. So it requires for efficiently completion of once-through operation in sampling period, which requires relatively low computational complexity [7-8]. To realize this objective, Suykens[2] has proposed online learning algorithm using Multi-mode constraints, OLAMMC, which can convert multi-mode constraints learning issues to solution by linear system of equations so as to improve the arithmetic speed. This article mainly uses multi-mode constraints learning model to achieve the incremental and online learning algorithm using multi-mode constraints.

\section{Android smartphone based English learning platform using multi-mode constraints}

Major difference between multi-mode constraints and standard multi-mode constraints is that, through the latter, inequality constraints can successfully be converted into equality constraints so as to make empirical risk deviation as a quadratic form. Specific multi-mode constraints formulation is as below:

Assume training dataset is given: 


$$
\left(x_{i}, y_{i}\right), i=1,2, \ldots, l, x_{i} \in R^{n}, y_{i} \in R \text {, }
$$

Set

$$
y(x)=w^{T} \phi(x)+b
$$

This linear function to fit sample set, use nonlinear mapping $\phi(\cdot)$, data of inpu space can be mapped into characteristic space so as to convert original nonlinear problem into more maneuverable linear fitting problem. After comprehensively considering fitting errors, structural risk and other factors, following constrained optimization form can be expressed as a regression problem:

$$
\begin{gathered}
\min _{w, b, e} J(w, e)=\frac{1}{2} w^{T} w+\frac{C}{2} \sum_{i-1}^{l} e_{i}^{2}, \\
\text { s.t. } y_{i}=w^{T} \phi\left(x_{i}\right)+b+e_{i}, i=1,2, \ldots, l
\end{gathered}
$$

To solve the problem, it requires for further adjustment of constraint optimization problem. Construct Lagrange as below:

$$
L(w, b, e, \alpha)=J(w, e)-\sum_{i=1}^{l} \alpha_{i}\left\{w^{T} \phi\left(x_{i}\right)+b+e_{i}-y_{i}\right\}
$$

Obtain based on KKT condition:

$$
\left\{\begin{array}{l}
\frac{\partial L}{\partial w}=0 \rightarrow w=\sum_{i=1}^{l} \alpha_{i} \phi\left(x_{i}\right) \\
\frac{\partial L}{\partial b}=0 \rightarrow \sum_{i-1}^{l} \alpha_{i}=0 \\
\frac{\partial L}{\partial e_{i}}=0 \rightarrow \alpha_{i}=C e_{i} \\
\frac{\partial L}{\partial \alpha_{i}}=0 \rightarrow w^{T} \phi\left(x_{i}\right)+b+e_{i}-y_{i}=0
\end{array}\right.
$$

After eliminating $e_{i}, w$ from formula (3), obtain:

$$
\left[\begin{array}{cc}
0 & e 1^{T} \\
e 1 & Q+C^{-1} L
\end{array}\right]\left[\begin{array}{l}
b \\
\alpha
\end{array}\right]=\left[\begin{array}{l}
0 \\
y
\end{array}\right]
$$

Among all,

$$
\begin{array}{r}
y=\left(y_{1}, y_{2}, \ldots, y_{l}\right)^{T}, e 1=(1,1, \ldots, 1)^{T}, \\
\alpha=\left(\alpha_{1}, \alpha_{2}, \ldots, \alpha_{l}\right)^{T}, \\
Q_{i j}=\left(\phi\left(x_{i}\right) \cdot \phi\left(x_{j}\right)\right)=k\left(x_{i}, x_{j}\right), i, j=1,2, \ldots, l .
\end{array}
$$

fitting function obtained, i.e. imput of multi-mode constraints is:

$$
y(x)=\sum_{i=1}^{l} \alpha_{i} k\left(x, x_{i}\right)+b
$$

It can be obtained from analysis expression (4) that, multi-mode constraints training problems ca be successfully converted into solution by linear equation. Compared with standard multi-mode constraints, it does not require for solution of complex quadric form programming problem, but 
only to solve problems by simpler linear system of equations.

Compared with standard multi-mode constraints, multi-mode constraints have following characteristics: (1) multi-mode constraints has smaller computational complexity; (2) solution of multi-mode constraints is more imagination supportive, possible for whole training samples, thus the explanation lack sparsity; (3) multi-mode constraints can effectively unify multi-mode constraints, bays technology, and gaussian process so as to analyze the essential relations between each; (4) multi-mode constraints can expand dynamic problems to autoregression for solution.

\section{Simulation experiment}

This paper examines the learning ability of the algorithm in English, Duffing:

$$
\begin{array}{r}
\dot{x}_{1}=x_{2} \\
\dot{x}_{2}=-p_{1} X_{1}-p_{2} X_{2}-x_{1}^{3}+q \cos \left(w t_{1}\right),
\end{array}
$$

English motivated learning behavior will occur when $p_{1}=-1.1, p_{2}=0.4, q=1.6, w=1.8 . t_{1}$ is the continuous variable of time.

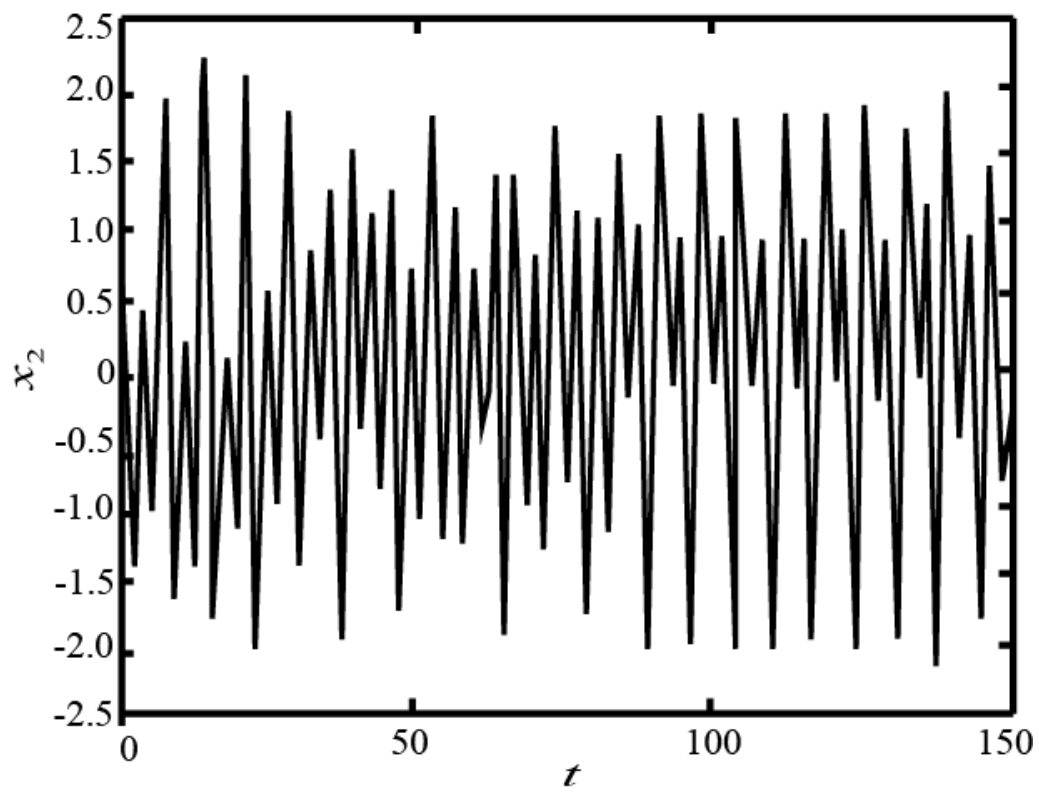

Fig. 1. Chaotic behavior under the state of ${ }^{X_{2}}$

Conduct discretization processing on Duffing equation, to obtain:

$$
x_{2}(t)=f\left(x_{2}(t-1), x_{1}(t-1), t_{1}(t)\right),
$$

Structure of learning sample can be realized: $x_{t}=\left(x_{2}(t-1), x_{1}(t-1), t_{1}(t)\right), y_{t}=x_{2}(t)$. Firstly conduct simulation research on incremental algorithm. Through sample $\left(x_{t}, y_{t}\right)$ obtain sample $\operatorname{set}\left(x_{t}, y_{t}\right)_{t-1}^{N}$. Then, use sample set to carry out training on incremental algorithm. In this simulation experiment, regard Android smartphone based college English learning platform as an offline learning algorithm. Based on features of this algorithm, the previous iterative operation result can be well applied. Thus it has computational complexity. Through simulation experiment and comparative analysis based on OLAMMC Android smartphone based college English learning platform and OLAMMC learning method, obtain results shown in figure 1: 
Table 1. Comparison between OLAMMC incremental algorithm and OLAMMC direct arithmetic for inverse system

\begin{tabular}{|c|c|c|c|c|}
\hline & \multicolumn{2}{|c|}{ run time $(\mathrm{s})$} & \multicolumn{2}{c|}{ test error (\%) } \\
\hline $\begin{array}{c}\text { Number of training } \\
\text { sample set }\end{array}$ & $\begin{array}{c}\text { Incremental } \\
\text { algorithm }\end{array}$ & $\begin{array}{c}\text { Direct arithmetic for } \\
\text { inverse system }\end{array}$ & Incremental algorithm & $\begin{array}{c}\text { Direct arithmetic for } \\
\text { inverse system }\end{array}$ \\
\hline 100 & 0.5320 & 1.3600 & 0.1802 & 0.1517 \\
\hline 200 & 1.8760 & 4.9860 & 0.1408 & 0.1307 \\
\hline 300 & 6.0540 & 12.1710 & 0.1250 & 0.1217 \\
\hline 400 & 11.6580 & 20.3450 & 0.1082 & 0.1058 \\
\hline 500 & 18.4860 & 33.4250 & 0.0905 & 0.0786 \\
\hline
\end{tabular}

It can be obtained from analysis results that two algorithms have no big differences in learning accuracy but have large differences in run time. In comparison, OLAMMC incremental algorithm requires shorter run time.

Next, study online learning algorithm through simulation experiments. Similarly, with sample $\left(x_{t}, y_{t}\right)$ obtain window type sample set with window length of 2, i.e. $x(t)=\left[x_{t}, x_{t-1}\right], y(t)=\left(y_{t}, y_{t-1}\right)^{T}, t=1,2 \ldots$. Then use sample set for online algorithm training. Compare and analyze OLAMMC based online learning algorithm and MMC based online learning algorithm. Figure 2 and figure 3 are specific simulation results:
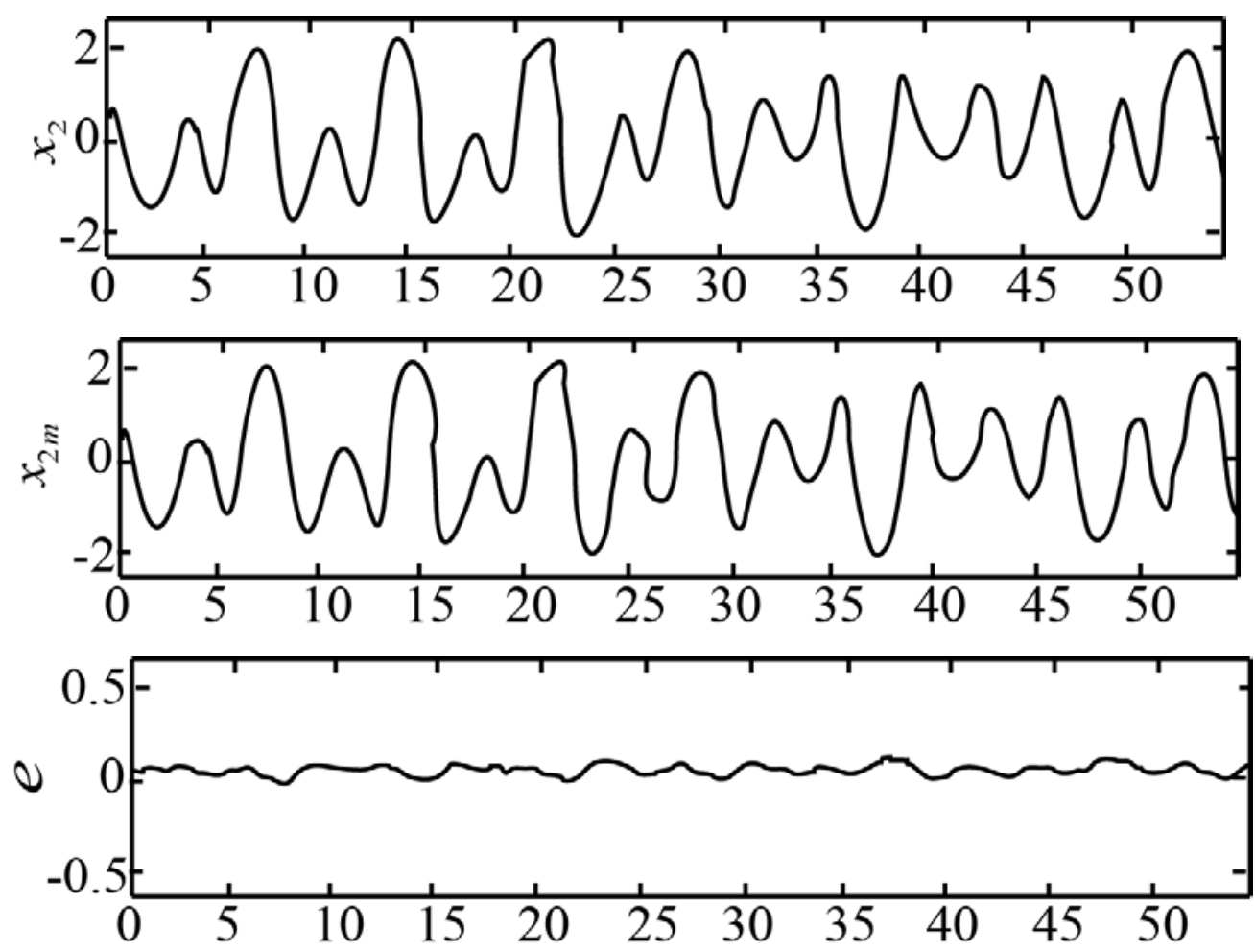

Fig. 2. Simulation result of OLAMMC based college English learning 

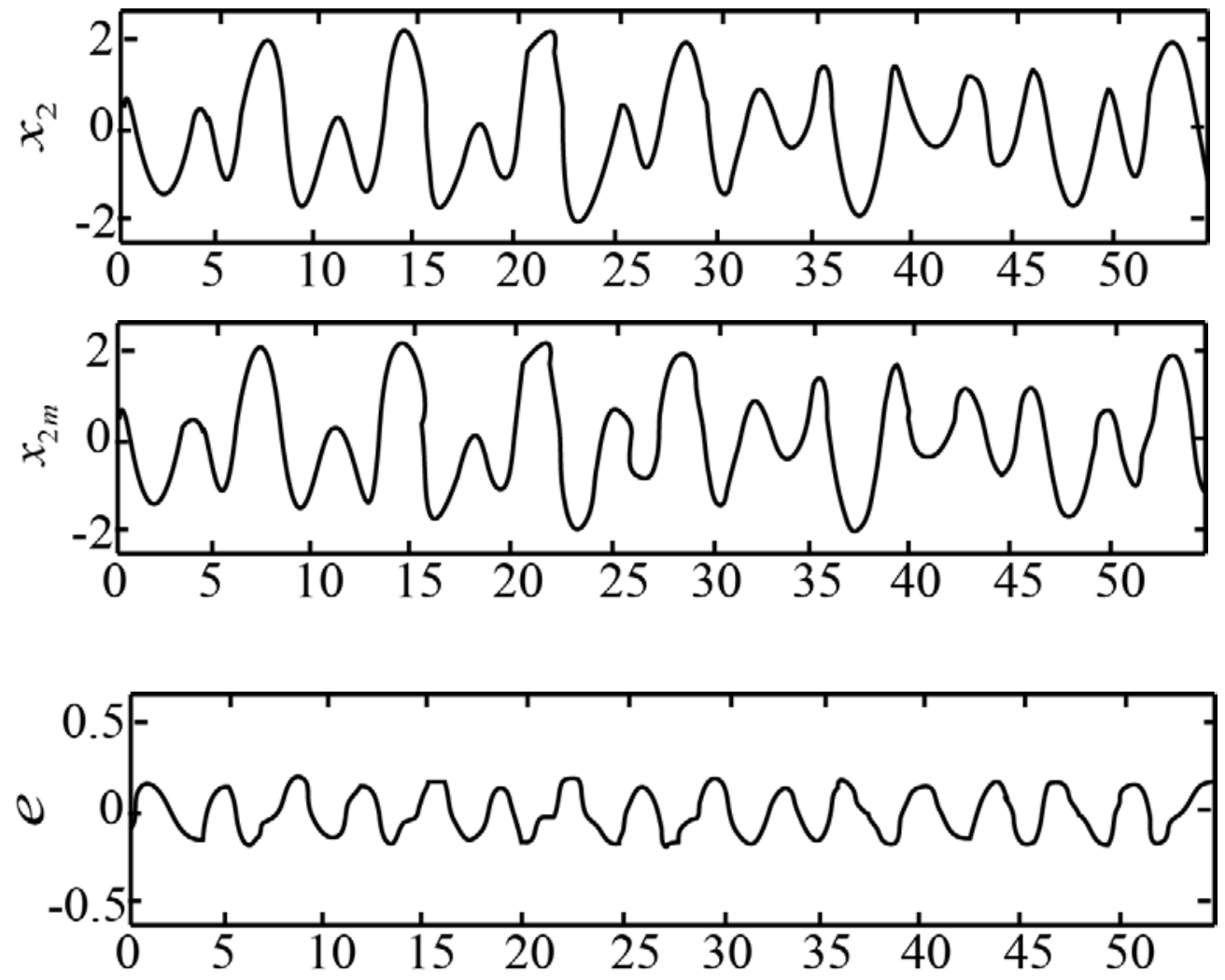

Fig. 3. Simulation result of MMC based college English learning

It can be obtained that, OLAMMC based college English learning designed in this article can realize effective English learning. This article has also fully verified the traceability and learning performance of the algorithm. Results show that it is better than MMC based college English learning platform in both two aspects.

\section{Conclusion}

This article has firstly conducted feature analysis on mathematical model using multi-mode constrains, and then implemented Android smartphone based college English learning platform using multi-mode constraints by means of kernel function,s characteristics and block matrix, and also fully verified its performance through simulation experiments.

\section{Acknowledgement}

Funded scientific research projects: 2016 Jiangxi Provincial Department of Education Science and Technology Project: "based on the Android smartphone college English mobile learning platform design and development" one of the research results, Number: GJJ160849.

\section{Reference}

[1] G es te l T. V., Suykens J. A. K., Lanckriet G. . Bayesian framework for least squares support vecto $\mathrm{r}$ machine classifiers Gaussian processes and kernel fisher discriminant analysis. Neural Computation, 2002, 15(5): 1115 -1148.

[2] Suykens J. A.K., Vandewalle J. . Recurrent least squares sup port vector machines. IEEE Transactions on Circuits and Systems, 2000 47(7): 1109 -1114.

[3] Joachims T. . Text Categorization with support vector ma chines: Learning with Many Relevant Features. In: Proceedings of the European Conference on Machine Learning( EGML),1998, 
$137-142$.

[4] eston J., Barnhill S. . Gene selection for cancer classification using support vector machines. Machine Learning, 2002, 46( 1): 389 -422.

[5] Kivinen J., Smola A., illiams on R. . Online learning with kernels. In: Die 11 rich T. G., Becker S., GhahramaniZ. ed s.. Advances in N eural Information Processing System s. Cambridge MA: MIT Press, 2002, 785 -793.

[6] Ralaivola L. Incremental support vector machine learning :A local approach. in: Proceedings of the International on Conference on Artificial Neural Networks Vienna Austria, 2001, 322 -329.

[7] Ruping S. Incremental learning with support vector machines. Dortmund L niversity, Dortmund: T echnical Report T R, 18, 2002.

[8] Martin M. . On line support vector machines for function approximation. Politecnica L niversity, Catalunya Spain: Tech nical Report LSI, 02,11 R, 2002. 